\title{
Reflets
}

Revue d'intervention sociale et communautaire

\section{Francophones et Premières Nations : le regard politique d'un ancien travailleur social}

\section{Entrevue avec Bernard Richard}

\section{Hélène Albert et François Boudreau}

Volume 22, numéro 2, automne 2016

La recherche et l'intervention en travail social en contexte

francophone minoritaire au Canada

URI : https://id.erudit.org/iderudit/1038969ar

DOI : https://doi.org/10.7202/1038969ar

Aller au sommaire du numéro

Éditeur(s)

Reflets, Revue d'intervention sociale et communautaire

ISSN

1203-4576 (imprimé)

1712-8498 (numérique)

Découvrir la revue

Citer ce document

Albert, H. \& Boudreau, F. (2016). Francophones et Premières Nations : le regard politique d'un ancien travailleur social : entrevue avec Bernard Richard.

Reflets, 22(2), 20-42. https://doi.org/10.7202/1038969ar d'utilisation que vous pouvez consulter en ligne.

https://apropos.erudit.org/fr/usagers/politique-dutilisation/ 


\section{Francophones et Premières Nations : le regard politique d'un ancien travailleur social}

\section{Entrevue avec Bernard Richard}

Avocat et ancien travailleur social, Bernard Richard a été député à l'Assemblée législative du Nouveau-Brunswick et a été nommé ombudsman et premier Défenseur des enfants et de la jeunesse du Nouveau-Brunswick. Il est expert dans les domaines des droits de la personne, des droits de l'enfant, de la justice sociale et des inégalités qui existent dans la société canadienne. Depuis quatre ans, il s'intéresse surtout aux droits de l'enfant en ce qui concerne la santé mentale (Access Canada, Partners for Youth, Xchange NB), le développement international (Plan Canada) et les enfants autochtones (First Nations Children's Futures Fund). Monsieur Richard appuie présentement le travail d'un regroupement de Chefs autochtones dans les domaines de la protection de l'enfance et la réforme de l'assistance sociale.

Cette entrevue, réalisée en janvier 2016 par Hélène Albert et François Boudreau, présente une réflexion sur des enjeux liés aux réalités des francophones en contexte minoritaire et à celles des communautés autochtones au Canada. Si, pour ces groupes minoritaires, les défis sont nombreux et complexes — et les luttes toujours nécessaires

— il semble qu'il faille demeurer optimistes, vigilants et engagés!

Reflets: Monsieur Richard, nous savons que vous êtes impliqué dans le projet "Access : esprits ouverts, open minds ", organisme qui développe la recherche transformationnelle sur la santé mentale des adolescents. Pouvez-vous nous expliquer en quoi l'accès aux services dans la langue du requérant à partir de ses paramètres culturels est important?

Bernard Richard : Access : esprits ouverts, open minds est un projet national piloté par des chercheurs de l'Université McGill, qui a été mis en œuvre dans douze sites à travers le Canada. Certains des sites 
fonctionnent en français, d'autres en anglais et, dans la province du Nouveau-Brunswick, le site fonctionne dans les deux langues. Il y a également des sites qui exercent leurs activités dans des communautés autochtones, par exemple à Eskasoni (CapBreton) et au Nunavik, une communauté inuite dans le Grand Nord du Québec. Donc, c'est un projet de recherche qui se déroule principalement en anglais. Il y a la traduction simultanée pour les rencontres générales, mais la majorité des participants sont anglophones. Sur les sites cependant, le travail se fait dans les deux langues, selon les besoins des requérants. Il y a deux sites à Montréal; là, c'est surtout en français que se déroulent les interventions. Les langues et les cultures sont une variable importante dans le projet. Cela est important parce que, selon mon expérience et selon le consensus social à ce propos, en plus d'être des éléments clés pour établir une relation de confiance, la langue et la culture sont des déterminants sociaux de santé. Permettre aux gens qui cherchent notre appui, nos services et notre aide de le faire dans leur langue et dans un climat culturel confortable et respectueux, aide ces requérants à nous faire confiance et contribue à les rendre également beaucoup plus participatifs. Et ça, c'est absolument essentiel. Si on n'arrive pas à faire cela dans nos communautés, bien on aura des gens qui vont hésiter avant d'aller chercher des services. Il y en a aussi qui ne vont pas participer aussi pleinement, aussi ouvertement. La langue et la culture sont des éléments centraux dans tout processus social et individuel d'appartenance et de rattachement au groupe.

Reflets: Ily a donc deux dimensions à la question : la dimension de l'individu et la dimension sociale. Peut-elle également être politique?

Bernard Richard: Il faut répondre oui. Sur la dimension sociale qui rejoint la dimension politique, je peux mentionner un exemple. Il y a un débat aujourd'hui au Nouveau-Brunswick à propos des services ambulanciers, soit de savoir s'ils doivent être bilingues, dans le sens de la nécessité d'avoir au minimum un ambulancier bilingue dans chaque ambulance. Car vous savez que le Nouveau- 
Brunswick est officiellement bilingue. Cette question a créé une importante controverse dans la section des commentaires sur le site Internet de la CBC qui rapportait la nouvelle et dans les lettres à l'éditeur des journaux anglophones du NouveauBrunswick. On pouvait lire de très nombreux commentaires selon lesquels une telle politique enlèverait des jobs aux anglophones, parce que forcément ça va être des francophones qui vont avoir ces jobs-là, ce sont eux qui sont bilingues. Mais la question de fond est une question de service; que ce soit à Caraquet ou à St. Stephen, quelqu'un qui a besoin d'un service ambulancier doit pouvoir l'obtenir dans sa langue. Ce n'est pas juste une question de droit, c'est une question de santé, de se faire comprendre, d'expliquer son mal, etc. C'est vrai qu'à Caraquet, il n'y a pas beaucoup d'anglophones, et qu'à St. Stephen ou à McAdam, il n'y a pas beaucoup de francophones, mais c'est absolument nécessaire que ces services soient disponibles parce qu'on ne sait jamais à qui on va avoir affaire et quels seront leurs besoins. Puis, parfois, en fonction de l'âge, ou dans une situation traumatique, ou en cas d'accident, tu peux perdre ta langue seconde assez rapidement...

Dans une situation de crise, ta langue, c'est essentiel, tu penses dans ta langue. Mais, hélas, c'est un débat assez courant au Nouveau-Brunswick de nos jours, comme beaucoup d'autres aspects de la question linguistique.

Reflets: Nous constatons encore que ce sont surtout les anglophones qui se sentent menacés par le bilinguisme. Est-ce parce qu'ils sont proportionnellement moins nombreux à être bilingues que les francophones?

Bernard Richard : On a vu à quel point, avec la controverse entourant le cas de madame d'Entremont, la commissaire aux langues officielles, à qui un agent de sécurité n'a pu répondre en français, puisque c'est une langue qu'il ne comprend pas. Il travaillait à l'entrée de l'édifice qui abrite le bureau du premier ministre. Un agent 
unilingue anglais... Cela a fait couler beaucoup d'encre, parce que la commissaire a décidé d'enquêter sur sa propre expérience.

Ce n'est pas nécessairement une situation idéale, mais cela indique que la question linguistique demeure non réglée. Après beaucoup d'efforts et beaucoup d'années, après presque cinquante ans de politique de langues officielles, on est toujours en plein cœur de ce débat-là. Il semble que cela dérange encore les anglophones, parce que leur histoire est différente. Leur histoire s'est développée dans une province où la langue majoritaire était dominante, et on ne se posait pas ce genre de questions jusqu'aux années 1960. La question était rarement posée, rarement réfléchie. C’est certain que le bilinguisme officiel, la dualité linguistique au ministère de l'Éducation, l'enchâssement des droits dans la Constitution, tout cela a changé la donne, puis ça dérange certaines personnes. Je crois toujours que la majorité de la population appuie le bilinguisme officiel au Nouveau-Brunswick, mais c'est certain que ça dérange encore certaines personnes.

Reflets : En tant que formatrices et formateurs de travailleuses et travailleurs sociaux, nous nous intéressons naturellement beaucoup à la question du bien-être social. Pour vous, qu'est-ce que ça signifie le bien-être social?

Bernard Richard : Bien, pour moi, le bien-être se traduit par un mot : le bonheur; par le fait de se sentir bien dans sa peau et dans sa communauté. Quand je réfléchis à ces questions, je me dis que c'est aussi la possibilité de vivre dans une société qui permet à chacun d'atteindre son plein potentiel. Peu importe ce potentiel. Donc, ce n'est pas la même chose pour tout le monde, mais il est essentiel que la possibilité d'atteindre son potentiel puisse être la même pour tout le monde. Pour moi, vivre dans une société où il y a de l'équité, ça veut dire de permettre aux gens de poursuivre leurs rêves, peu importe quels sont ces rêves. Évidemment, cela doit toujours se faire dans le respect des rêves des autres. Notre liberté s'arrête là où commence celle des autres. C'est pourquoi je m’identifie, je me suis toujours identifié au programme de 
chances égales pour tous de Louis Robichaud (ancien premier ministre du Nouveau-Brunswick). On a souvent dit qu'au Nouveau-Brunswick, le programme de chances égales de Louis Robichaud, c'était pour les Acadiens. Mais, dans le fond, ce n'était pas du tout cela. C'était surtout pour les régions rurales, où il y avait moins d'argent, moins de revenus d'impôt, des écoles sans gymnase, comme à Cap-Pelé, où j'ai grandi, tandis que, dans les plus grandes villes, toutes les écoles avaient des gymnases, même des piscines dans certains cas, parce qu'il y avait des ressources beaucoup plus importantes. Et le programme de chances égales a effectivement égalisé les chances pour tout le monde en redistribuant l'argent de manière plus équitable. Il est certain que ce programme politique a soulevé d'énormes débats. J'ai vu des articles dans le journal de Fredericton, avec des titres comme " Robbing Peter to pay Pierre », vous imaginez? «To pay Pierre $»$ !

Il y a eu les débats dans les années 1960 dans The Daily Gleaner, un journal qui, avant la fusion des journaux appartenant à la famille Irving, était très loyaliste et qui tenait ce discours, alors que Louis Robichaud était premier ministre, soit le premier premier ministre acadien élu dans une élection générale. Les anglophones voyaient cela comme une menace à leurs acquis, mais la portée du programme, qui était d'une importance capitale pour nous, il faut bien l'admettre, faisait aussi l'affaire des anglophones vivant en milieu rural.

Pour revenir au bien-être social, parce que c'est votre question, pour moi ça signifie que tous les citoyens et toutes les citoyennes ont la possibilité d'être heureux. Même dans la Constitution américaine, on parle de "right to happiness " (Life, Liberty and the pursuit of Happiness). Alors, pour moi, c'est quelque chose de fondamental pour tout être humain. Cela ne veut pas dire le droit de porter des armes ouvertement, car, là, c'est autre chose. Je ne ferais pas l'éloge de toute la Constitution américaine, mais je trouve que c'est intéressant. Je trouve que c'est un aspect intéressant de la Constitution américaine qu'on dise que la 
poursuite du bonheur est un droit fondamental, un droit qui doit être protégé. Donc, pour moi, c’est un peu ça, une société bienveillante qui gère ses programmes pour assurer l'égalité des chances pour tous.

Reflets: Donc, le droit au bonheur, ou la poursuite du bonheur, n'est pas simplement un élan individuel vers son propre bonheur, mais ça serait aussi un élan qui doit être soutenu par une collectivité?

Bernard Richard : Oui, c'est un bonheur collectif autant qu'un bonheur individuel. Cette dimension collective est celle du rôle de l'État. C'est ce que je vois comme la dimension interventionniste de l'État. En politique, je crois beaucoup dans le rôle de l'État; autrement, la société est un endroit du chacun pour soi, et on sait ce que ça donne. C'est certain qu'on a besoin d'un équilibre dans tout cela, car il y a eu des tentatives de créer des sociétés tout à fait égalitaires, et ça ne fonctionne pas non plus. Donc, il faut trouver un équilibre juste. Ce travail, un peu comme celui qu'exige la protection de la langue d'une minorité, c'est un travail qui n'est jamais terminé.

Reflets: Vous avez parlé du premier ministre Robichaud, qui développait des programmes de redistribution des richesses pour favoriser l'équivalence des conditions. Il y a eu au Canada ce qu'on a appelé, comme partout en Occident, l'État providence. Nous nous sommes un peu éloignés de cela depuis quelques décennies. Aujourd'hui, la situation de la richesse se caractérise par sa polarisation. Au Canada, le $1 \%$ de la population la plus riche contrôle à peu près $15 \%$ de la richesse, et les $10 \%$ des plus riches contrôlent à peu près $30 \%$ de la richesse. À l'échelle internationale, le $1 \%$ des plus riches contrôle à peu près $50 \%$ de la richesse. Pensez-vous que nous sommes rendus à la fin de ce mouvement de balancier? Pensez-vous que nous commençons à voir des signes qui nous permettraient de revenir à une situation un peu plus équilibrée, ou bien pensez-vous que nous ne sommes pas encore rendus à un retour d'un équilibre plus grand ou plus équitable? 
Bernard Richard : J'espère que nous sommes rendus à un retour des choses, enfin je le pense. Je prends comme exemple le succès de Bernie Sanders, qui est un des candidats à l'investiture démocrate aux ÉtatsUnis et qui fait une forte campagne sur ce thème de la polarisation des richesses. Il se décrit haut et fort comme socialiste. Aux États-Unis, il faut le faire là. Et puis il mène une très chaude lutte à Hillary Clinton. Tout l'establishment du parti appuie très fortement la femme de l'ancien président Bill Clinton, mais le socialiste Sanders est en tête en Iowa et au New Hampshire. Cela ne fera pas nécessairement de lui le candidat démocrate aux prochaines élections américaines, mais c'est intéressant parce qu'il est quand même à l'extrême de ce point de vue des inégalités, qui sont encore plus fortes aux États-Unis qu'au Canada. Aux États-Unis, certains mènent une bataille farouche pour être libres d'à peu près toute forme d'impôt. Au Canada, les inégalités sont, pour l'instant, moins fortes qu'aux ÉtatsUnis, quoique nous nous soyons rapprochés des Américains sur cette question à l'époque du gouvernement de Stephen Harper, et même avant. Mais j'ai l'impression qu'avec le nouveau gouvernement Trudeau, il y a eu une réaction très opposée à ce genre d'approche, à la politique du chacun pour soi. De toute manière, cette approche ne fonctionne pas; elle crée des sociétés où il y a énormément de criminalité. Les États-Unis sont la société la plus riche au monde, alors qu'en même temps, ils sont la société où il y a le plus haut taux d'incarcération au monde. Cependant, dans certains États américains, y compris au Texas, on commence à réaliser qu'en emprisonnant tout le monde pour de très longues périodes, on exacerbe la situation sociale dans plusieurs communautés. Cette société de polarisation des richesses est également la société ayant le taux de criminalité et le taux de possession d'armes le plus élevé au monde, et les tragédies comme on a vu se multiplient. Plus d'un incident tragique par arme à feu par jour aux ÉtatsUnis en moyenne, impliquant plusieurs victimes, soit plus de trois victimes par incident. Beaucoup d'Américains réalisent qu'ils sont allés trop loin. Certainement, au Canada, nous venons de vivre une 
course vers le bas. Ce phénomène de la réduction des impôts à tout prix... Je crois savoir de quoi je parle, j'ai travaillé avec un premier ministre qui était très à cheval làdessus, qu'on appelait le meilleur premier ministre conservateur, même s'il était un libéral. L'idée était qu'en réduisant massivement les impôts, cela produirait une richesse économique qui serait partagée par tout le monde, le soi-disant trickle down economics, mis de l'avant par Ronald Reagan et Margaret Thatcher au début des années 1980. Mais ça ne fonctionne pas cette théorie. Je demeure convaincu que ce n'est pas une politique qui fonctionne. Il faut trouver l'équilibre et, pour revenir au mouvement de balancier, j'espère qu'on est arrivé à la fin de ce mouvement (vers la droite). Il y a aussi eu des sociétés où l'exagération est inverse, par exemple la Grèce, où la retraite se prenait encore récemment à l'âge de $52 \mathrm{ou}$ 53 ans, où d'autres peuvent facilement éviter de payer de l'impôt, un fait connu et longtemps toléré. Il faut encourager le sens des responsabilités au sein de la population, il y a un équilibre à trouver et à maintenir. Cet équilibre devrait viser le bien-être, c'est-à-dire donner une chance égale à tout le monde, protéger les plus vulnérables, et ainsi de suite. Mais si l'État va trop loin, certains développent le sentiment que l'État est l'ennemi de la liberté, puis ils vont faire tout ce qu'ils peuvent pour éviter de prendre leurs responsabilités, pour ne pas payer d'impôt, et là se développe une économie souterraine plus importante (le travail au noir). Quand les gens perçoivent l'État comme étant l'ennemi, un voleur presque qui vient leur prendre leur gagnepain, et bien ils vont faire ce qu'ils pensent qu'ils doivent faire pour éviter de payer de l'impôt puis, dans ces conditions, c'est tout le monde qui perd à la fin. Donc, c'est pour cela, à mon avis, que c'est important de trouver l'équilibre.

Le modèle le plus proche de l'idéal que j'ai vu, c'est celui des pays scandinaves. Quand on regarde les études qui se font sur l'indice du bonheur, études qui se font depuis quelques années, les populations scandinaves arrivent toujours en tête de classement de l'indice du bonheur. Pour moi, cette donnée est 
très importante, parce que justement elle reflète des sociétés où les taux d'imposition sont quand même assez élevés, mais où le filet social est très important aussi. J'ai l'impression qu'on a trouvé là un modèle qui fonctionne, la preuve est que les gens y sont heureux. Ce n'est pas un paradoxe; les sociétés les plus taxées au monde sont des sociétés où les gens sont le plus heureux. Pour moi, ça veut dire quelque chose.

Reflets : Observez-vous également des écarts plus grands de bien-être social selon la composition culturelle des populations au Canada?

Bernard Richard: C'est bien évident, surtout à partir de mon travail avec les communautés autochtones. Je travaille beaucoup dans deux dossiers importants : la réforme ou la restructuration des agences de protection de l'enfance et la réforme de l'assistance sociale. Je suis souvent dans les communautés autochtones et $\mathrm{j}$ 'ai aussi des rencontres avec les chefs des communautés autochtones du Nouveau-Brunswick. Là, c'est évident que la situation de la pauvreté est d'un autre ordre que ce que nous trouvons dans les communautés non autochtones. Il y a probablement une multitude de raisons pour cela. De plus, les Autochtones qui vivent à l'extérieur de leurs communautés, en milieu urbain, à Winnipeg, à Edmonton, à Calgary, à Vancouver, ont très souvent, certainement de façon disproportionnée, besoin de services sociaux et ils vivent dans la pauvreté. En ce qui concerne les francophones, et j'ai probablement voyagé sur toutes les routes du NouveauBrunswick, j'ai vu des coins où il y avait de la pauvreté. Tant dans les régions anglophones que dans les régions francophones. Je pense que le programme de chances égales a finalement réussi à équilibrer les choses. Les grandes disparités qui existaient au NouveauBrunswick, certainement, ont été amoindries. D'un point de vue culturel, c'est très remarquable par rapport aux Autochtones surtout.

Des statistiques publiées à l'automne 2015 sur les taux de pauvreté infantile indiquent que les taux les plus élevés au Canada sont observés à Toronto et à Saint-Jean au Nouveau- 
Brunswick. Puis Saint-Jean vient de dépasser Toronto. Il n'y a pas si longtemps, des dix communautés les plus pauvres au Canada, il y en avait six qui étaient des Premières Nations du Nouveau-Brunswick, selon un document publié par Statistique Canada. Ça m’avait frappé énormément. Je ne connais pas de milieu épargné par la pauvreté.

Reflets : Comment se manifestent ces écarts d'accès aux richesses?

Bernard Richard: Ce qui s'observe, c'est la qualité du logement, le fait que des familles partagent les logements. Dans un dossier qui m’intéresse, la réforme de l'assistance sociale dans les communautés autochtones, le fédéral veut imposer les taux de chaque province dans les communautés autochtones qui se trouvent dans une province donnée. En principe, ça semble avoir du sens, sauf qu’il $\mathrm{y}$ a des provinces où certains programmes permettent d'ajouter $\mathrm{du}$ soutien financier aux prestations d'assistance sociale. Ces programmes n'existent pas dans les communautés autochtones, ce qui crée un manque à gagner, une disparité pour les familles autochtones dans ces conditions.

Reflets: Vous parlez, par exemple, des suppléments pour les frais de chauffage et de choses du genre?

Bernard Richard : Oui, les suppléments au chauffage ou les programmes de transition vers le travail ou vers la formation. Il y a des programmes qui sont gérés par les provinces et où le financement est partagé entre le fédéral et la province. Ces programmes mis en œuvre par des ministères provinciaux, ou par des organismes qui sont financés par la province, n'existent généralement pas ou existent à un moindre niveau dans les communautés autochtones. Par exemple, dans le système d'aide sociale au Nouveau-Brunswick, le ministère travaille avec le concept d'unité économique. L'unité économique désigne une unité formée de personnes qui vivent sous un même toit. Bien, dans les communautés autochtones, ce n'est pas rare que deux ou trois familles partagent un même logement. En pratique, sous le système provincial, considérer 
cette maisonnée comme une unité économique aurait pour effet de diminuer l'assistance reçue.

Dans la plupart des communautés autochtones, les maisons appartiennent à la communauté, pas aux individus. La terre appartient toujours à la communauté. Il y a des exceptions en ce qui concerne les maisons, mais, dans la plupart des communautés autochtones, les maisons appartiennent à la communauté. Les hypothèques sont donc émises au nom de la communauté, de même que les prêts pour construire et réparer les logements. Sous le système provincial, l'aide sociale va directement à la famille, pas à la communauté. Les gouvernements autochtones sont très préoccupés par cela, puisque cela les forcera à aller chercher les loyers des résidents, ce qui change considérablement leurs manières de faire. Puis souvent, les familles sont dysfonctionnelles, ce qui complique encore les choses. Il y a donc toutes sortes de problèmes à considérer. En fin de compte, les personnes responsables de ces programmes dans les communautés autochtones pensent que ce sera bien difficile dans plusieurs cas de récupérer les loyers, et les communautés vont être aux prises avec des paiements d'hypothèques. Le ministère des Affaires autochtones semble tout à fait déterminé à procéder à cette réforme, sans tenir compte des énormes différences culturelles et de l'impact sur les familles, les individus et les communautés.

\section{Reflets : Et l'impact du nouveau gouvernement Trudeau?}

Bernard Richard: Il y a de très bonnes déclarations, le début du mandat est très encourageant, mais les attentes sont très élevées. J'étais à une réunion de chefs mi'kmaq du Nouveau-Brunswick, à Halifax, parce qu'il y avait une réunion des Premières Nations de l'Atlantique qui s'y tenait le lendemain. Ce qu'ils disaient à cette réunion, c'est qu'ils ont eu de très bonnes rencontres avec la ministre des Affaires autochtones, madame Carolyn Bennett, avec la ministre de la Justice, madame Jody Wilson-Raybould, qui est Autochtone, et qu'ils sont très encouragés. La ministre 
Bennett leur a fait savoir, juste avant Noël, de ne pas trop s'en faire à propos de la réforme de l'assistance sociale, qu'elle allait repousser la date d'un an et que le nouveau gouvernement fédéral n'avait pas l'intention de poursuivre cette réforme, mais, d'un point de vue technique, le plus qui pouvait être fait rapidement pour le moment, c'était de repousser la réforme d'un an. Il reste que le son de cloche des fonctionnaires régionaux n'a pas changé au même rythme, pas encore.

Par rapport à l'ancien gouvernement, donc, les attentes sont très élevées. Mais il faudra être patient, car, entre la volonté politique et les attentes de la population, il y a la bureaucratie, c'est-à-dire la machine d'État. J'ai connu cela de première main en ma qualité de ministre et de membre de conseil des ministres. Il y a souvent une marge qui crée un espace de temps entre la volonté et sa réalisation, c'est aussi cela la culture politique. On verra ce que ça donnera. Le premier ministre lui-même a assisté à des rencontres. Il a dit de bonnes choses, il indique vouloir réparer les relations avec les Premières Nations, qui sont en très mauvais état certainement. Mais le leadership autochtone est très encouragé. Il est trop tôt pour le dire, mais le ton a changé pour le mieux; c'est déjà du progrès.

Reflets : La question du racisme historique, nous pourrions dire "structurel" envers les Premières Nations, est-elle arrivée à un point tournant? Est-ce que les Canadiens commencent à prendre conscience de l'importance de mieux ressaisir leur relation historique avec les Premières Nations?

Bernard Richard : Oui. Le travail du juge Sinclair de la Commission de vérité et réconciliation a ouvert beaucoup d'esprits. Moi je disais souvent à la blague, " elle risque d'être forte en vérité, mais pas très forte en réconciliation ". Mais la Commission a entendu des témoignages terribles, qu'on a pu lire lors de la publication du premier sommaire en juin 2015. Par exemple, nous avons appris qu'on avait plus de risques de décéder si on était un enfant dans un pensionnat autochtone que si on était un soldat canadien 
durant la Deuxième Guerre mondiale. Les pensionnats avaient des cimetières, mais pas de gymnases. Souvent les enfants étaient enterrés sans pierres tombales, sans indications de qui ils étaient. C'est certain que le racisme a été très fort envers les Autochtones, et il existe toujours. Le leadership antiraciste doit venir d'en haut, du gouvernement. Il y a eu cet exemple inverse d'un ancien ministre des Affaires autochtones et du Nord, qui a dit quelque chose de grossier, et qui m'a beaucoup choqué; il a dit que les jeunes Autochtones sont impatients d'avoir 18 ans pour avoir leurs propres chèques d'assistance sociale. Quand on entend quelque chose comme cela de la bouche du ministre responsable du dossier, ce n'est pas facile d'établir des relations qui soient saines et constructives. Les paroles du nouveau gouvernement vont certainement davantage dans le bon sens, mais il faudra passer à l'acte. Ça va coûter très cher, parce qu'au fil des ans on a créé des situations désastreuses. D'un autre côté, l'ironie reste que la population qui croît le plus au Canada, c'est la population autochtone.

Il y a des jeunes, il y en a beaucoup, parce que le taux de natalité est aujourd'hui très important au sein des populations autochtones, en passant... On dit qu'on a besoin d'attirer des immigrants, et on a besoin de le faire. Mais on a toute une population autochtone qu'on abandonne là. Il est temps qu'on parle du syndrome d'alcoolisation foetal, à peu près 20 fois plus important dans les communautés autochtones que dans les communautés non autochtones. La surreprésentation dans le système carcéral, dans le système de justice pénale, et les taux de dépendance aux drogues, aux médicaments, et quoi d'autre? Donc, c'est certain qu'il y a énormément de travail à faire auprès des populations autochtones pour remédier au racisme historique, structurel, à leur exclusion systématique, à leur marginalisation et à l'impact des pensionnats. Cela ne se fera pas du jour au lendemain, mais ça commence par ça; ça commence par une volonté politique. Et cela, c'est aussi nouveau pour les communautés autochtones. Ce travail prendra aussi un appui de 
la population canadienne en général. Surtout quand l'économie va mal, dans les périodes d'austérité. C'est durant ces périodes que les gens sont plus sensibles par rapport à la protection de leurs acquis, et la population a alors tendance à réagir en demandant : "Pourquoi vous investissez là, mais pas chez nous? Pourquoi vous fermez mon école, mais vous investissez dans les communautés autochtones?»

Et puis les gens mélangent souvent toutes les formules et les sources de financement, entre le fédéral, le provincial et le municipal. Mais surtout dans des périodes d'austérité, il est important que les gens aient une bonne compréhension de notre responsabilité historique de réparer les torts qui ont été causés aux populations autochtones, ces dommages faits largement par nous. Je pense que la situation change lentement, mais sûrement. Je l'espère en tout cas.

Reflets : Monsieur Richard, vous avez eu une carrière assez riche d'expériences diverses. Vous avez été organisateur communautaire, politicien, fonctionnaire, ombudsman, défenseur des enfants et de la jeunesse, et même un modèle pour plusieurs, tout cela dans la seule province qui est officiellement bilingue au Canada. Vous avez aussi été à l'origine du poste de commissaire aux langues officielles du NouveauBrunswick, et vous avez produit un rapport dans le but de vous assurer que ce poste, comme les autres postes d'agents de l'Assemblée législative, demeure non partisan. D'un point de vue professionnel, au terme de tous les efforts déployés, est-ce que ça marche?

Bernard Richard : Est-ce que ça marche? Bien, on en a parlé un petit peu plus tôt, et je dirais que c'est un travail qui ne sera jamais complété. Nous vivons dans un monde où la langue anglaise prend de plus en plus de place. Nous vivons à côté non seulement d'une puissance économique, qui fait que les affaires se passent en anglais, mais aussi d'une puissance culturelle, les États-Unis. Les gens font leurs sélections de culture, de musique, de cinéma, de télévision à partir des choix qui sont offerts, et c'est la même chose partout 
dans le monde, mais nous, nous sommes juste à côté. Donc, c'est clair que cette puissance a un impact. En plus d'être minoritaires chez nous, nous sommes minoritaires sur le continent. Et peu importe où l'on se trouve dans le monde, même en France, le poids culturel de notre voisin se fait sentir.

Bon, j'aime taquiner mes amis français quand ils disent le week-end, le parking, le shopping et tout le reste. Pour eux, ça fait chic, mais ils ne sont pas menacés comme nous, pas de la même manière. Pour nous, c'est absolument essentiel de nous donner toutes les protections que nous puissions nous donner, et il faut être extrêmement vigilant de ce côté-là. C'est pour cela que j'ai été très, très, très surpris et même déçu de tout le débat qui a entouré la commissaire aux langues officielles l'automne dernier (2015). Quand on pense que le premier ministre a remis en question l'existence même du poste, que le ministre responsable de la révision des programmes a dit que " tout était sur la table ", y compris le poste de la commissaire aux langues officielles, quand le ministre responsable des langues officielles - qui en principe est le premier ministre selon la loi, mais, dans les faits, c'est le ministre de l'Énergie et des Mines qui a la responsabilité des langues officielles ici - quand ce ministre a dit que c'était une nomination politique, au sens de partisane, j'ai été estomaqué. C'est peut-être la nomination la moins partisane dans l'histoire du Nouveau-Brunswick, une nomination accompagnée d'un processus de sélection rigoureux auquel a participé à l'époque l'actuel premier ministre... Ce n'était pas du tout partisan. Et ça adonne que le premier ministre et ces deux ministres sont tous les trois des Acadiens, imaginez mon étonnement de les entendre dire que " tout est sur la table».

Malheureusement, dans ce dossier, le premier ministre a réagi politiquement, au lieu de réagir en homme d'État. À mon avis, le premier ministre a manqué à sa responsabilité de protéger les lois et les droits acquis des populations, peu importe de qui il s'agit. La réaction m’a énormément déçu. 
Il faut avoir à la fois le courage de ses convictions, même si je reconnais que ce n'est pas toujours facile. Quand j'étais député, c'était plus difficile pour moi aussi de prendre position comme ça. Lors de mon premier mandat, l'Association francophone des municipalités du Nouveau-Brunswick avait invité Lucien Bouchard, alors chef de l'opposition à Ottawa et chef du Bloc Québécois, comme conférencier à son assemblée annuelle. Le gouvernement d'alors - dont je faisais partie en tant que député d'arrière-ban et dont Frank McKenna était le premier ministre - avait décidé de boycotter cet événement qui recevait monsieur Bouchard. Il avait été décidé que le ministre des Affaires municipales n'y participerait pas. Comme ça se passait à Shediac, dans ma circonscription, l'Association m'avait invité pour y dire quelques mots de bienvenue. À ma grande surprise, le bureau du premier ministre a tout fait pour ne pas que j'y aille. Ils m'ont proposé d'aller donner une conférence ailleurs, à Alliance Québec, la même fin de semaine que la conférence de monsieur Bouchard!

Après des jours de tiraillement, j'ai quand même décidé de me rendre à l'assemblée des municipalités du Nouveau-Brunswick, à Shediac. L'événement se déroulait dans ma circonscription, là où se trouve la plus belle plage au Canada, la plage Parlee, où des milliers de Québécois viennent chaque année. J'ai souhaité la bienvenue à monsieur Bouchard, comme je l'aurais fait pour n'importe quel Québécois qui vient chez nous, sans nécessairement partager ses opinions politiques et puis ça, ça s'est bien passé. Sauf que certains de mes partisans politiques disaient alors " tu ne seras jamais nommé ministre. Tu ne peux pas t'opposer au premier ministre ». Puis, en plus, McKenna était connu comme quelqu'un qui menait d'une main de fer. Ce fut donc une décision extrêmement difficile pour moi, surtout que je n'avais pas l'indépendance que j'ai maintenant; je reconnais que j'ai de la chance. C'est dire à quel point la discipline de parti peut brimer la liberté d'expression d'un député. 
J'ai vécu des émotions semblables lors de l'adoption de la nouvelle Loi sur les langues officielles en 2002, l'œuvre de Bernard Lord, premier ministre conservateur. Il a très habilement joué son jeu en invitant l'ancien premier ministre, Louis Robichaud, à appuyer la réforme, à venir prendre la parole à l'Assemblée législative et à s'asseoir dans le siège du premier ministre. Cela ne s'était jamais fait!

Mais le projet de loi qu'il proposait et qui créait pour la première fois un poste de commissaire aux langues officielles - que j'appuyais en principe - manquait certains éléments clés, comme l'offre active et la responsabilité de promotion de la loi, comme c'est le cas dans la loi fédérale. J'avais proposé des amendements qui déplaisaient à mon chef, qui venait tout juste d'être élu à la tête du parti. Un anglophone très bien pensant, il appuyait le projet de loi, mais il était mal à l'aise avec l'idée de faire un débat sur cette question-là, le bilinguisme. J'ai dû insister très fort et j'ai trouvé l'expérience très lourde et déplaisante, parce que je voulais m'aligner avec mon chef.

Finalement, j'ai décidé de foncer. On a les outils qu'on a et donc je m'étais présenté à l'Assemblée législative avec le rapport Guérette-Smith ${ }^{1}$, le rapport Bastarache-Poirier ${ }^{2}$ et d'autres rapports et $j$ ' avais dit au premier ministre Lord, avant l'ouverture de la session, " ça peut prendre 10 minutes, ou ça peut prendre 10 jours, mais il faudra s'entendre sur des amendements, des modifications au projet de loi ». Il a organisé un petit caucus dans l'antichambre du gouvernement avec quelques ministres, puis ils ont finalement accepté mes amendements et les ont incorporés dans le projet de loi. Il y a des outils qui existent, mais ce n'est pas toujours facile de s'en servir. Les taux d'assimilation sont toujours préoccupants; les francophones ont déjà constitué presque $34 \%$ de la population du Nouveau-Brunswick, nous sommes maintenant à peine plus de $30 \%, 31 \%$. Les nouveaux arrivants, ce n'est pas de leur faute, mais ils adoptent l'anglais dans une très haute proportion. Nous n'aurons jamais trop d'outils à notre disposition. 
J'ai justement entendu une entrevue avec le ministre Dominic LeBlanc, qui disait " on ne peut pas vraiment agir à partir des langues officielles dans le cas des réfugiés syriens, on réagit à une situation d'urgence ». Il faut bien sûr tenir compte des situations concrètes, mais on dit souvent ça : "On peut rien faire. " On dit souvent ça. Si nous continuons à attirer des immigrants qui, dans un pourcentage de 80 à $85 \%$, sont anglophones ou adoptent la langue anglaise, bien la proportion de francophones va continuer à diminuer. Tous ces éléments sont importants à considérer. Il faut être très vigilant. C'est sûr que, parce que nous sommes minoritaires, c'est notre lot d'être vigilants. La situation ne sera jamais simple ni facile pour nous.

Je suis récemment allé visiter mon fils, qui vit en ColombieBritannique. De passage à Richmond, juste à l'extérieur de Vancouver, j'ai été surpris de voir le nombre de panneaux commerciaux qui étaient dans deux langues, sauf que c'était l'anglais et le mandarin, partout. Le Canada est un très grand pays, qui doit pouvoir protéger des droits acquis, qui sont aussi des droits constitutionnels. Notre culture et notre langue devront faire l'objet d'une grande vigilance. Nous avons de la chance au Nouveau-Brunswick, nous sommes quand même plus de $30 \%$ de la population. Ça compte pour quelque chose, même si nous avons la fâcheuse habitude de tenir pour acquis des droits si durement obtenus. Notre nombre nous donne quand même une force politique, ça nous donne des communautés qui fonctionnent presque entièrement en français, puis ça nous a donné des lois et la dualité au ministère de l'Éducation. Nous avons des acquis que d'autres minorités n'ont pas. Mais, si cette proportion diminue, ce sera autre chose. Il ne faut pas aller trop loin, juste traverser le pont de la Confédération, à l'île-du-PrinceÉdouard, pour constater que, même si les francophones font des efforts importants, le taux d'assimilation, qui est ici autour de $11 \%$, dépasse $40 \%$ là-bas. Et en Ontario, c’est la même chose, avec un taux de 40 à $50 \%$. Je ne veux pas m'inquiéter le ventre plein, mais, quand même, il faut comprendre qu'il est possible de perdre nos acquis si on ne s'en occupe pas. 
Reflets: Ce qu'on comprend, c'est qu'il faut continuer à se battre, mais est-ce que ça se fait sans avoir des alliés de l'autre communauté linguistique? Parce que, un petit peu à l'instar des Autochtones, qui ont mené toutes ces batailles qui ont donné lieu à la Commission de vérité et réconciliation, est-ce que ça ne prend pas aussi des alliés dans la communauté pour maintenir et défendre les acquis, pour assurer une pérennité de la culture et de la langue? Dans le même sens, sur la même question, est-ce que ça marche? Cinquante ans après la Commission Laurendeau-Dunton ${ }^{3}$, est-ce que ça marche le bilinguisme, le biculturalisme? Est-ce qu'on la voit cette génération suivante qui est convaincue de ce que la génération précédente a voulu léguer?

Bernard Richard : Oui. En fait, si on examine les programmes d'immersion, on voit qu'il y a un certain engouement pour ces programmes. J'ai eu à diriger des bureaux, j'ai engagé des jeunes anglophones bilingues, et de très bons bilingues. C'est certain qu'il y a toujours des défis; même au Nouveau-Brunswick, on a un peu reculé, on a abandonné le programme d'immersion précoce et, pour moi, ça a été une erreur monumentale dans la seule province officiellement bilingue du Canada. Il fallait apporter des améliorations, mais le fait de l'enlever a été un recul; ce n'était pas une bonne décision, et j'ai honte de dire que c'est un gouvernement libéral qui a pris cette décision. En ce qui concerne le biculturalisme, le Canada n'a jamais été biculturel. Multiculturel, oui, il y a des Ukrainiens, des Iraniens, des Chinois, des Vietnamiens. C'est bien clair quand on va à Toronto, par exemple, on voit des communautés de partout dans le monde. C'est une ville extrêmement multiculturelle. Montréal aussi, mais Toronto davantage, je dirais. Pour moi, le biculturalisme n'a jamais existé, seul le bilinguisme officiel existe. Laurendeau-Dunton a créé ou entretenu une certaine fiction qui n'a jamais existé comme telle au Canada, parce que le Canada, c'est énorme, et puis l'Ouest canadien est différent, loin de la culture française. Mais, ce que cette commission nous a donné, c'est davantage de ressources, des programmes de langues officielles, des appuis aux communautés de langues officielles, 
des appuis aux minorités, même là où les francophones sont très minoritaires dans certains cas. Finalement, là où le nombre le justifie, ce rapport a été l'enclenchement d'une réforme qui nous a donné des écoles. Donc, pour moi, la Commission a lancé une époque très riche et importante pour la minorité francophone.

Nous pouvons parler d'un bilinguisme officiel et très certainement d'un multiculturalisme qui va continuer. Le Canada va continuer à se diversifier dans les années à venir, parce que notre taux de natalité ne nous permet pas de maintenir notre niveau de vie et, donc, il faudra compter sur les immigrants. Et d'ailleurs on en attire 300000 par année, sans compter le programme de réfugiés; ce sont de très grands nombres. Puis, on n'a pas le choix de le faire, ça va donner un Canada beaucoup plus multiculturel qu'il ne l'est déjà. Il l'est déjà pas mal. Ça se voit peut-être moins au Nouveau-Brunswick, mais ça se voit très certainement à Vancouver, à Toronto, à Montréal, puis dans d'autres villes. À la fin, ça ne veut pas dire qu'on ne peut pas exister comme pays officiellement bilingue, mais il va falloir travailler très fort pour maintenir le niveau de bilinguisme, les droits qu'on a et la langue française en particulier. À mon avis, elle est menacée à long terme, pas à court terme, pas à moyen terme, mais à long terme. Si on n'arrive pas à prendre les moyens. Le chercheur Rodrigue Landry à l'Université de Moncton parle d'autonomie culturelle. Pour moi, c'est plus réaliste. On pourrait penser une province acadienne virtuelle, par exemple.

Reflets: Je vais me permettre une petite parenthèse sur ce qui relève de l'actualité. À la suite des événements concernant Marcel Aubut et le Comité olympique canadien, un rapport a été préparé dans les deux langues. Bien le rapport en français, même sur sa page titre, était bourré de fautes. Au-delà de l'intention bienveillante, ily a peut-être aussi le comment on assume la langue, sa qualité. Nous en sommes là. Je me rappelle aussi un autre événement. Je ne veux pas faire un long aparté, mais je devais rédiger une lettre de recommandation pour le Barreau du Nouveau-Brunswick, et le gabarit qu'on mavait 
envoyé pour mindiquer ce que je devais y consigner était dans un français très approximatif. Je me suis dit, ça c'est un organisme qui gère le droit dans une province bilingue, une province qui enseigne le français, la common law en français, et je reçois un document qui est bourré de fautes! Il y a l'idée du bilinguisme, et il y a la réalité du bilinguisme. La qualité de la langue, le niveau de langue, comment est-ce qu'on assure un niveau minimum de qualité? Cela m’apparaît important.

Bernard Richard : Je suis d'avis que tout cela passe par le système d'éducation puis par le développement de la petite enfance. Dès la petite enfance, il faut des programmes de qualité de formation des maîtres, puis des enseignantes et des enseignants hautement qualifiés, puis l'accès à des outils culturels en français. Je comprends qu'il y a des organismes privés comme le Barreau - un organisme privé, pas public - qui demeurent assez maladroits à répondre aux exigences des deux langues officielles au Nouveau-Brunswick, même si je dois reconnaitre qu'ils y travaillent de plus en plus. D'un autre côté, je regarde mes petits-enfants, qui fréquentent les écoles francophones du Nouveau-Brunswick, et je regarde la qualité de leur français, le matériel auquel ils ont accès. (Je sais aussi que dans certains cas, comme à Moncton, il y a beaucoup d'anglais qui se parle dans la cour d'école. Tout cela est complexe, parce qu'on a aussi l'obligation d'accepter les ayants droit. Puis, dans certaines écoles, ils sont assez nombreux. Et, à Moncton, par exemple, ils vivent dans un milieu où l'anglais est dominant.) Je me suis égaré un peu. Je disais que j'écoute parler mes petitsenfants et j'essaie de me rappeler, ça fait longtemps, mais j'essaie de me souvenir de moi, à cet âge-là, et je trouve qu'on a quand même fait des progrès importants. En tout cas, j'aime croire qu'on en a fait. Les produits culturels auxquels ils ont accès sont beaucoup plus nombreux, et la qualité de ces produits est impressionnante. Quand j'étais adolescent, dans les années 1960, j'écoutais à peu près exclusivement de la musique anglaise : Jimmy Hendrix, les Beatles, etc., mais maintenant l'offre du produit culturel en français s'est beaucoup développée. Je dirais 
que, pour moi, tout a commencé avec le groupe de musique 1755 et peut-être Garolou et Harmonium. Mais aujourd'hui, il y a une très grande variété et une grande quantité de matériel pédagogique, mais aussi culturel : des livres, du théâtre, etc. Donc, je considère qu'on a avancé. Ce n'est pas négligeable. En même temps, je regarde mon téléphone portable et Internet et tout, et là, ça se passe beaucoup trop en anglais, y compris pour nos jeunes, qui commencent à se promener avec des tablettes dès l'âge de 10 ans. Tout cela a l'air d'une nouvelle menace à la langue et à la culture, qui sert à nous rappeler encore une fois que notre travail ne sera jamais terminé.

Reflets : Monsieur Richard, merci beaucoup pour votre générosité!

\section{Notes}

1 «En mars 1986, le rapport Guérette-Smith, intitulé Rapport du Comité consultatif sur les langues officielles du Nouveau-Brunswick, est publié. Ce rapport contient les opinions de la population quant à l'usage des deux langues officielles ainsi que les conclusions du comité. Il présente également des données comparatives sur le nombre d'employés anglophones et francophones au sein de la fonction publique entre 1985, 1982 et 1978."

http://www.languesofficielles.nb.ca/publications-liens-et-autres/histoire-des-langues-officielles

2 «En mars 1982 est publié le rapport Poirier-Bastarache du groupe de travail du Nouveau-Brunswick sur les langues officielles, Vers l'égalité des langues officielles au Nouveau-Brunswick. Celui-ci fait état des résultats d'une étude menée en vue de la révision de la Loi sur les langues officielles de 1969. Le rapport présente des données sociolinguistiques et démographiques du Nouveau-Brunswick, ainsi que de l'information détaillée sur le nombre d'employés anglophones et francophones qui travaillent dans les services publics. "

http://www.languesofficielles.nb.ca/publications-liens-et-autres/histoire-des-langues-officielles

3 "Commission royale dienquête sur le bilinguisme et le biculturalisme. L'une des commissions ayant eu le plus d'influence sur le cours de l'histoire canadienne, la Commission royale d'enquête sur le bilinguisme et le biculturalisme (1963-1969) change radicalement la politique linguistique aux échelles fédérale et provinciales. Elle est créée en réaction au malaise grandissant parmi les Canadiens français du Québec qui demandent la protection de leur langue et de leur culture, et attendent lıccasion de participer pleinement à la prise de décisions en matière politique et économique. Les conclusions de la commission mènent à des changements en matière d'éducation 
en français partout au pays, à la création du ministère fédéral du Multiculturalisme et à l'adoption de la Loi sur les langues officielles".

http://www.encyclopediecanadienne.ca/fr/article/commission-royale-denquete-sur-le-bilinguismeet-le-biculturalisme/ 